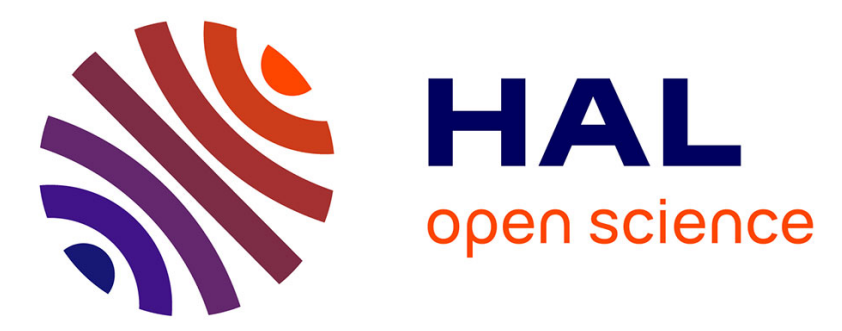

\title{
Parametric resonance-induced time-convolutionless master equation breakdown in finite size exciton-phonon systems
}

Vincent J.C. Pouthier

\section{- To cite this version:}

Vincent J.C. Pouthier. Parametric resonance-induced time-convolutionless master equation breakdown in finite size exciton-phonon systems. Journal of Physics: Condensed Matter, 2010, 22 (38), pp.385401. 10.1088/0953-8984/22/38/385401 . hal-00522013

\section{HAL Id: hal-00522013 \\ https://hal.science/hal-00522013}

Submitted on 29 Sep 2010

HAL is a multi-disciplinary open access archive for the deposit and dissemination of scientific research documents, whether they are published or not. The documents may come from teaching and research institutions in France or abroad, or from public or private research centers.
L'archive ouverte pluridisciplinaire HAL, est destinée au dépôt et à la diffusion de documents scientifiques de niveau recherche, publiés ou non, émanant des établissements d'enseignement et de recherche français ou étrangers, des laboratoires publics ou privés. 


\title{
Parametric resonance-induced time-convolutionless master equation breakdown in finite size exciton-phonon systems.
}

\author{
Vincent Pouthier \\ Institut UTINAM, Université de Franche-Comté, \\ UMR CNRS 6213, 25030 Besançon cedex, France. \\ E-mail: vincent.pouthier@univ-fcomte.fr
}

\begin{abstract}
A detailed analysis is performed to show that the second order timeconvolutionless master equation fails to describe the exciton-phonon dynamics in a finite size lattice. To proceed, special attention is paid to characterize the coherences of the exciton reduced density matrix. These specific elements measure the ability of the exciton to develop superimpositions involving the vacuum and the one-exciton states. It is shown that the coherences behave as wave functions whose dynamics is governed by a time-dependent effective Hamiltonian defined in terms of the so-called time-dependent relaxation operator. Due to the confinement, quantum recurrences provide to the relaxation operator an almost periodic nature so that the master equation reduces to a linear system of differential equations with almost periodic coefficients. In accordance with the Floquet theory, we show that unstable solutions emerge due to parametric resonances involving specific frequencies of the relaxation operator and specific excitonic eigenfrequencies. These resonances give rise to an unphysical exponential growth of the coherences indicating the breakdown of the second order master equation.
\end{abstract}




\section{Introduction}

In molecular lattices and large molecules, excitons are ideal candidates to carry energy from one place to another and to activate specific chemical reactions. For instance, amide-I excitons that promote vibrational energy flow in proteins are expected to play a key role in the transduction of the chemical energy into mechanical work $[1,2,3,4,5,6,7,8,9,10,11]$. Similarly, Frenkel excitons that delocalize along the array of pigment proteins in light-harvesting complex allow the conversion of solar energy into chemical energy $[12,13,14,15,16]$. Finally, vibrons in adsorbed molecular nanostructures provide an alternative to nanoscale electronics and may enhance surface reactions $[17,18,19,20,21,22,23,24]$. The common feature between the latter examples is that molecular lattices exhibit regularly distributed atomic subunits. Mainly due to dipole-dipole interactions, the energy associated to a specific electronic transition, or to a high frequency vibrational mode, delocalizes between these subunits. It gives rise to a narrow-band exciton able to move coherently along the lattice. Unfortunately, the exciton does not propagate freely but it interacts with the vibrations of the host medium usually described by phonons [25, 26]. The phonons induce stochastic fluctuations of the dynamical parameters that control the exciton dynamics and they tend to destroy the coherent nature of the exciton.

The problem so defined is rather general and it refers to a quantum open system (exciton) coupled with a thermal bath (phonons) $[27,28,29]$. In that case, the information required to describe the exciton properties is encoded in the exciton reduced density matrix $(\mathrm{RDM})$. It is extracted from the density matrix of the whole system via a partial trace over the phonon degrees of freedom. From the Liouville-von Neumann equation, the aim of the theory of open systems is to derive a generalized master equation (GME) to characterize the RDM time evolution. To proceed, the projector method plays a central role. It consists in eliminating the irrelevant information connected to the bath to extract the information that is desired to derive the GME. Therefore, two different kinds of GME have been obtained. In the NakajimaZwanzig approach [30, 31], the GME is an integro-differential equation that involves the time convolution (TC-GME) between the RDM and the memory kernel. By contrast, in the Shibata approach [32,33], a different resummation of the perturbation series is performed to obtain the so-called time-convolutionless master equation (TCLGME). The TCL-GME being local in time, the influence of the bath is encoded in a time-dependent relaxation operator. Unfortunately, although the projector method yields an elegant way to derive an exact GME, it is only formal and the solution of the GME still requires the solution of the full problem. However, the projector method provides a starting point for the derivation of approximate GME through a systematic perturbation expansion with respect to the system-bath interaction.

In many situations, narrow-band excitons propagate slower than the phonons and the nonadiabatic weak coupling limit is reached. The Born approximation is invoked and a second order perturbation expansion is performed to derive a $\mathrm{TCL}_{2}$-GME or a $\mathrm{TC}_{2}$-GME. Therefore, the kernel memory and the time-dependent relaxation operator are expressed in terms of the exciton-phonon coupling correlation functions. The dynamics of the correlation functions is governed by free phonons which do not experience the exciton and which behave as a reservoir [34]. Note that in the strong coupling limit, a similar procedure is used but within the polaron point of view, i.e. after performing a partial renormalization of the exciton-phonon interaction $[35,36,37,38]$. In that context, two situations occur depending on the size of the 
lattice. In an infinite lattice, the correlation functions vanish after a time scale of about the time needed to the phonons to cover a lattice site. This phonon correlation time being shorter than the time required to the exciton to propagate freely, the Markov limit is reached so that the $\mathrm{TC}_{2}$-GME and the $\mathrm{TCL}_{2}$-GME are equivalent [39]. They correspond to a system of first order differential equations with constant coefficients whose solution provides relevant information about the exciton properties (coherence transfer [10], diffusion coefficient [5, 38, 40], linear and nonlinear absorption spectra $[24] \ldots)$.

In a finite size lattice, a different behavior takes place due to the discrete nature of the phonon energy spectrum $[8,41,42,43]$. Because the phonons are confined, time recurrences and strong memory effects occur in the correlation functions. The memory kernel and the time-dependent relaxation operator do no longer converge in the long time limit giving rise to a strong non-Markovian regime. In that case, as in most situations involving non-Markovian effects, it seems preferable to use the $\mathrm{TCL}_{2}$-GME which presents the advantage of being local in time [27]. The $\mathrm{TCL}_{2^{-}}$ GME yields exact results for specific problems, such as the Kubo oscillator [32] and the finite size exciton-phonon system in the zero hopping constant limit [41], and it gives a better approximation to the exact solution than the corresponding $\mathrm{TC}_{2}$-GME. Nevertheless, there are situations for which the Born approximation completely fails to provide good estimates of the RDM evolution ( divergence in the long time limit, violation of the positivity ...). This is effectively what happens when the exciton is confined in a finite size lattice. To overcome this problem, different strategies have be elaborated. Examples among many are the correlated projection superoperators method $[44,45,46]$, the time-dependent projection-operator approach $[47,48,49]$ and the effective-mode representation of non-Markovian dynamics [50, 51].

In the present paper, we do not derive an additional formalism to treat nonMarkovian effects nor apply existing theories. Instead, we present a detailed study to understand why the $\mathrm{TCL}_{2}$-GME exhibits instabilities which, of course, have no physical meaning. To proceed, we shall consider the Fröhlich model that describes a narrow-band exciton coupled with acoustic phonons [25]. Following recent works $[9,10,41]$, we shall restrict our attention to specific elements of the RDM, called coherences, that measure the ability of the exciton to develop superimpositions involving the vacuum and the one-exciton states. Their dynamics is governed by a system of first order differential equations with time dependent coefficients and it will be shown that instabilities take place due to parametric resonances.

The paper is organized as follows. In section 2, the exciton-phonon Hamiltonian is described and the coherences are defined. Then, the $\mathrm{TCL}_{2}$-GME is established for describing the coherence dynamics. A numerical analysis is performed in section 3 where special attention is paid to characterize the occurrence of instabilities in the GME. These results are discussed and interpreted.

\section{Theoretical background}

\subsection{Model Hamiltonian}

Let us consider a finite size one-dimensional lattice that contains $N$ sites. Each site $m=1, \ldots, N$ is occupied by an atomic subunit whose internal dynamics is described by a two-level system. Let $|m\rangle$ denote the first excited state of the $m$ th two-level system and $\hbar e_{0}$ the corresponding energy. Note that the convention $\hbar=1$ will be 
used throughout this paper. The vacuum state $|\oslash\rangle$ describes all the two-level systems in their ground state. In the local basis $\{|m\rangle\}$, the exciton Hamiltonian is defined as

$$
H_{A}=\sum_{m=1}^{N} e_{0}|m\rangle\langle m|+\sum_{m=1}^{N-1} J[|m+1\rangle\langle m|+| m\rangle\langle m+1|],
$$

where $J$ is the exciton hopping constant. Due to the confinement, the one-exciton states are superimpositions of incident and reflected plane waves. A stationary regime takes place and the exciton eigenstates correspond to $N$ normal modes with quantized wave vectors $K_{k}=k \pi / L$, with $k=1, . ., N$ and $L=N+1$, expressed as

$$
|k\rangle=\sum_{m=1}^{N} \sqrt{\frac{2}{L}} \sin \left(\frac{k \pi m}{L}\right)|m\rangle .
$$

In the eigenbasis $\{|k\rangle\}$, the exciton Hamiltonian is rewritten in terms of the eigenfrequencies $\omega_{k}=e_{0}+2 J \cos (k \pi / L)$ as $H_{A}=\sum_{k=1}^{N} \omega_{k}|k\rangle\langle k|$.

The exciton interacts with acoustic phonons that describe the external motions of the lattice sites. The phonon Hamiltonian is defined as

$$
H_{B}=\sum_{m=1}^{N} \frac{p_{m}^{2}}{2 M}+\sum_{m=1}^{N-1} \frac{W}{2}\left(u_{m+1}-u_{m}\right)^{2}+\frac{W}{2}\left(u_{1}^{2}+u_{N}^{2}\right),
$$

where $M$ is the mass of the $m$ th site whose displacement and momentum are $u_{m}$ and $p_{m}$, respectively, and where $W$ is the force constant between neighboring sites. The last two terms in (3) account for the fixed boundary conditions. Confined phonons define $N$ normal modes with quantized wave vectors $q_{p}=p \pi / L$, with $p=1, . ., N$. The corresponding frequencies are $\Omega_{p}=\Omega_{c} \sin (p \pi / 2 L)$ where $\Omega_{c}=\sqrt{4 W / M}$. Within this normal mode representation, the phonon Hamiltonian is rewritten in terms of the phonon operators $a_{p}^{\dagger}$ and $a_{p}$ as $H_{B}=\sum_{p=1}^{N} \Omega_{p} a_{p}^{\dagger} a_{p}$.

According to the Fröhlich model, the exciton-phonon interaction results from a stochastic modulation of the internal energy of each two-level system by the external motions of the lattice. Within the local basis, the energy of the $x$ th two-level system exhibits random variations proportional to the lattice distortion $u_{m+1}-u_{m-1}$, with $u_{0}=u_{L}=0$. Therefore, in the normal mode representation the coupling Hamiltonian is defined as

$$
\Delta H=\sum_{m=1}^{N} \sum_{p=1}^{N} \Delta_{p m}\left(a_{p}^{\dagger}+a_{p}\right)|m\rangle\langle m|,
$$

where $\Delta_{p m}$ is the strength of the stochastic modulation of the $m$ th two-level energy induced by the $p$ th phonon mode. It is expressed in terms of the coupling strength $\Delta_{0}$ as

$$
\Delta_{p m}=2 \sqrt{\frac{2}{L}} \Delta_{0} \sqrt{\sin \left(\frac{p \pi}{2 L}\right)} \cos \left(\frac{p \pi}{2 L}\right) \cos \left(\frac{p \pi m}{L}\right) .
$$

Note that $\Delta_{0}$ defines the so-called small polaron binding energy $E_{B}$ according to the relation $E_{B}=2 \Delta_{0}^{2} / \Omega_{c}$.

Similarly, inserting (2) into (4) yields the expression of the exciton-phonon interaction in the exciton eigenbasis, as

$$
\Delta H=\sum_{k_{1}=1}^{N} \sum_{k_{2}=1}^{N} \sum_{p=1}^{N} \Delta_{p k_{1} k_{2}}\left(a_{p}^{\dagger}+a_{p}\right)\left|k_{1}\right\rangle\left\langle k_{2}\right| .
$$


The coupling strength $\Delta_{p k_{1} k_{2}}$ is defined as

$$
\Delta_{p k_{1} k_{2}}=\sqrt{\frac{2}{L}} \Delta_{0} \sqrt{\sin \left(\frac{p \pi}{2 L}\right)} \cos \left(\frac{p \pi}{2 L}\right) S_{p k_{1} k_{2}}
$$

where

$$
S_{p k_{1} k_{2}}=\delta_{p, k_{1}-k_{2}}+\delta_{p, k_{2}-k_{1}}-\delta_{p, k_{1}+k_{2}}-\delta_{p, 2 L-k_{1}-k_{2}} .
$$

In the eigenbasis, the exciton-phonon interaction is responsible for the exciton scattering from $\left|k_{1}\right\rangle$, with energy $\omega_{k_{1}}$, to $\left|k_{2}\right\rangle$ with energy $\omega_{k_{2}}$, via the exchange of a phonon $p$ with energy $\Omega_{p}$. The allowed transitions are specified by the selection rules $S_{p k_{1} k_{2}} \neq 0$ that generalize the concept of momentum conservation in a finite size lattice. At this step, let us mention that we shall restrict our attention to the nonadiabatic weak coupling limit in which the following relations are satisfied: $\omega_{0} \gg \Omega_{c}$ (high energy exciton), $4 J<\Omega_{c}$ (nonadiabatic limit) and $E_{B} \ll J$ (weak coupling limit). In that case, there is no resonance in the sense that scattering processes do not conserve the energy, i.e. $\omega_{k_{1}}-\omega_{k_{2}} \neq \pm \Omega_{p}[5]$.

The exciton-phonon dynamics is thus governed by the full Hamiltonian $H=H_{A}+$ $H_{B}+\Delta H$ which will be used to study the properties of the exciton RDM. Although the present model is rather general, it will be applied to situations in which the lattice temperature cannot be disregarded, being typically of about to or higher than the phonon cutoff frequency. The phonons are thus expected to behave as a reservoir in thermal equilibrium. Such situations are rather common and they correspond, for instance, to the vibron dynamics in $\alpha$-helices $[1,2,3,4,5,6,7,8,9,10,11]$. In that case, peptide units $\mathrm{H}-\mathrm{N}-\mathrm{C}=\mathrm{O}$ are regularly distributed and neighboring units are linked by a $\mathrm{H}$ bond. Each site contains an amide-I mode, i.e. a high frequency $\mathrm{C}=\mathrm{O}$ stretching vibration, that gives rise to a vibrational exciton which delocalizes along the lattice. The exciton interacts with acoustic phonons that correspond to the collective vibrations of the $\mathrm{H}$ bond network.

\subsection{Coherences and $T C L_{2}-G M E$}

Since $H$ is an exciton-number conserving Hamiltonian, the Hilbert space is partitioned into independent subspaces as $\mathcal{E}=\mathcal{E}_{0} \oplus \mathcal{E}_{1} \ldots$, where $\mathcal{E}_{v}$ is the $v$-exciton subspace. Consequently the RDM exhibits independent blocks that evolve in distinct Liouville spaces with various dimensions. To study the breakdown of the $\mathrm{TCL}_{2}$-GME in a simple way, we restrict our attention to specific elements of the RDM called coherences. These coherences measure the ability of the exciton to develop superimpositions involving the vacuum and the one-exciton states. They define a $N$-component column vector whose dynamics is restricted to a Liouville space with a reduced dimension equal to $N$. Therefore, as detailed in $[9,10,41]$, coherences switch on when the exciton-phonon system is brought in a configuration out of equilibrium. To proceed, the exciton is prepared in the superimposition $\left|\psi_{A}\right\rangle=c_{0}|\oslash\rangle+c_{1}\left|m_{0}\right\rangle$, with $\left|c_{0}\right|^{2}+\left|c_{1}\right|^{2}=1$. Note that the generalization to other situations involving eigenbasis vectors is straightforward. By contrast, the phonons are assumed to be in thermal equilibrium at temperature $T$. Their properties are encoded in the standard Boltzmann density matrix $\rho_{B}$ so that the initial state of the whole system is specified by the density matrix $\rho=\left|\psi_{A}\right\rangle\left\langle\psi_{A}\right| \otimes \rho_{B}$.

In that context, within the local basis, the coherences are the RDM matrix elements $\sigma(m, \oslash, t)$ that measure the coherence between the ground state and local one-exciton states. These elements, simply noted $\sigma_{m}(t)$, are defined as

$$
\sigma_{m}(t)=\left\langle m\left|\operatorname{Tr}_{B}\left[e^{-i H t} \rho e^{i H t}\right]\right| \oslash\right\rangle,
$$


where $\operatorname{Tr}_{B}$ denotes a partial trace over the phonon degrees of freedom and where $\sigma_{m}(0)=\delta_{m m_{0}} c_{1} c_{0}^{*}$. The coherence $\sigma_{m}(t)$ gives the ability of the exciton to develop a superimposition involving $|\oslash\rangle$ and $|x\rangle$ at time $t$, given that such a superimposition was initially created on the site $m_{0}$. It generalizes the concept of transition amplitude and it defines the probability amplitude to observe the exciton on site $m$ at time $t$ given that it was on site $m_{0}$ at $t=0$. The generalization arises through the fact that during the transition the exciton interacts with a phonon bath at finite temperature. Similarly, from (2), the coherences in the eigenbasis are defined as

$$
\hat{\sigma}_{k}(t)=\sum_{m=1}^{N} \sqrt{\frac{2}{L}} \sin \left(\frac{k \pi m}{L}\right) \sigma_{m}(t) .
$$

As previously, $\hat{\sigma}_{k}(t)$ defines the survival amplitude of the eigenstate $|k\rangle$ and it measures the ability of the exciton to maintain a quantum state which is a superimposition involving $|\oslash\rangle$ and $|k\rangle$. Note that to work with normalized objects at $t=0$ one introduces the reduced coherences $\Psi_{m}(t)=\sigma_{m}(t) / c_{1} c_{0}^{*}$ and $\hat{\Psi}_{k}(t)=\hat{\sigma}_{k}(t) / c_{1} c_{0}^{*}$. One thus obtains $\Psi_{m}(t=0)=\delta_{m m_{0}}$ and $\hat{\Psi}_{k}(t=0)=\sqrt{2 / L} \sin \left(k \pi m_{0} / L\right)$.

In that context, by applying the TCL projector method within the Born approximation, the $\mathrm{TCL}_{2}$-GME that governs the dynamics of the coherences can be established [27, 32, 33]. To proceed, one can either represent the GME in the local basis or in the eigenbasis. Although both approaches are equivalent, we have observed that the numerical integration of the GME is more efficient within the eigenbasis. Therefore, after performing straightforward algebraic manipulations, the $\mathrm{TCL}_{2}$-GME is expressed as

$$
i \frac{d}{d t} \hat{\Psi}_{k_{1}}(t)=\omega_{k_{1}} \hat{\Psi}_{k_{1}}(t)-i \sum_{k_{2}=1}^{N} \mathcal{J}\left(k_{1}, k_{2}, t\right) \hat{\Psi}_{k_{2}}(t),
$$

where the time-dependent relaxation operator $\mathcal{J}(t)$ is defined as

$$
\mathcal{J}\left(k_{1}, k_{2}, t\right)=\sum_{k=1}^{N} \sum_{p=1}^{N} \frac{E_{B} \Omega_{p}}{L} \sqrt{1-\left(\frac{\Omega_{p}}{\Omega_{c}}\right)^{2}} S_{p k_{1} k} S_{p k k_{2}} F_{p k k_{2}}(t) .
$$

The function $F(t)$ is written as

$$
\begin{aligned}
F_{p k k_{2}}(t) & =\frac{i n_{p}}{\omega_{k_{2}}-\omega_{k}+\Omega_{p}}\left[1-e^{i\left(\omega_{k_{2}}-\omega_{k}+\Omega_{p}\right) t}\right] \\
& +\frac{i\left(n_{p}+1\right)}{\omega_{k_{2}}-\omega_{k}-\Omega_{p}}\left[1-e^{i\left(\omega_{k_{2}}-\omega_{k}-\Omega_{p}\right) t}\right],
\end{aligned}
$$

where $n_{p}=\left(\exp \left(\Omega_{p} / k_{B} T\right)-1\right)^{-1}$ is the Bose-Einstein distribution connected to the $p$ th phonon mode, $k_{B}$ being the Boltzmann constant.

The GME (11) is isomorphic to the Schrodinger equation for an exciton moving on a finite size lattice. The reduced coherence $\hat{\Psi}_{k_{1}}(t)$ behaves as a wave function whose dynamics is described by a time-dependent non-hermitian effective Hamiltonian $\mathcal{H}(t)=H_{A}-i \mathcal{J}(t)$. The first term in the right-hand-side of (11) describes the free evolution under the influence of the exciton Hamiltonian $H_{A}$, only. By contrast, the effect of the phonons is encoded in the relaxation operator $\mathcal{J}(t)$ which breaks the unitary nature of the evolution. The origin of this time-reversal symmetry breaking is twofold. First, it arises due to the non vanishing value of the real part of $\mathcal{J}(t)$. Then, it is enhanced by the fact that the imaginary part of $\mathcal{J}(t)$ is a non-hermitian matrix. 


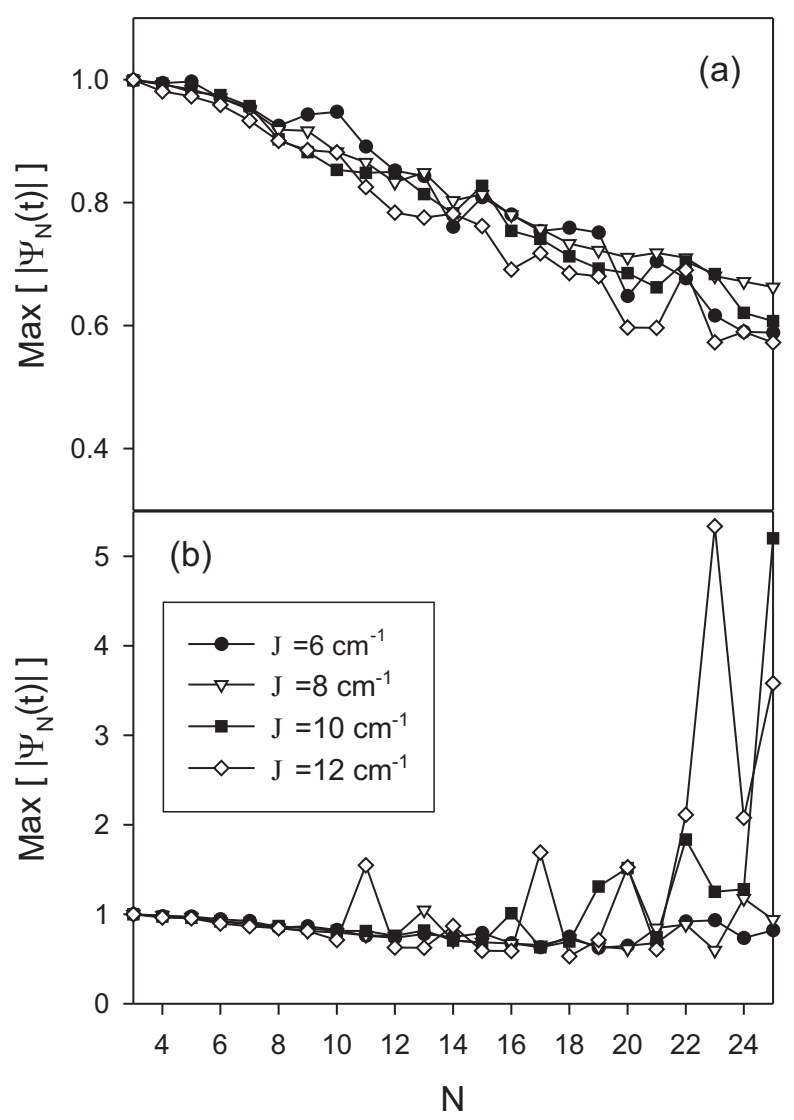

Figure 1. Maximum value of the transmitted coherence over 1000 ps versus lattice size for (a) $T=100 \mathrm{~K}$ and (b) $T=300 \mathrm{~K}$.

Therefore, equation (11) shows that the exciton-phonon interaction is responsible for a coupling between distinct coherences. Although this coupling is local in time, it results from the history of the interaction. Basically, a coupling takes place at time $t$ between $\left|k_{1}\right\rangle$ and $\left|k_{2}\right\rangle$ if $\left|k_{1}\right\rangle$ is scattered in a state $|k\rangle$ via the exchange of a phonon $p$ at time $t$ and if the phonon bath keeps the memory of a past interaction involving the scattering of $\left|k_{2}\right\rangle$ into $|k\rangle$ via the exchange of the same phonon $p$. Note that the selection rules encoded in the $\mathrm{S}$ matrix (see equation (8)) reveal that only coherences $\hat{\Psi}_{k_{1}}(t)$ and $\hat{\Psi}_{k_{2}}(t)$ that satisfy $\left|k_{1}-k_{2}\right|=2 q$, with $q=0,1,2, \ldots$, interact through the phonon bath.

\section{Results and Discussion}

In this section, the numerical integration of the $\mathrm{TCL}_{2}$-GME (11) is carried out to highlight the occurrence of instabilities for specific values of the model parameters. Following our recent work devoted to the coherence transfer in an infinite lattice [10], it will be applied to describe amide-I exciton in a finite size lattice of $\mathrm{H}$-bonded peptide units $[9,41]$. To proceed, typical values for the parameters are used: $e_{0}=1660$ 


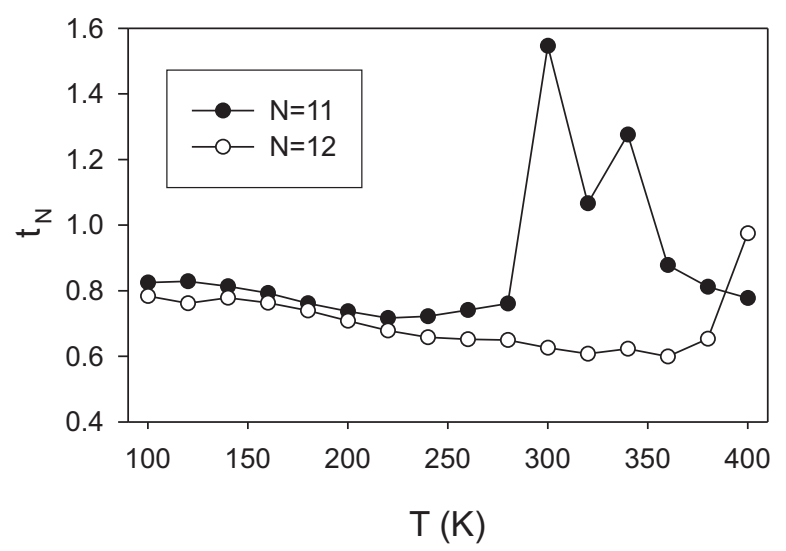

Figure 2. Maximum value of the transmitted coherence versus Temperature for $J=12 \mathrm{~cm}^{-1}, N=11$ (full circles) and $N=12$ (open circles).

$\mathrm{cm}^{-1}, W=15 \mathrm{Nm}^{-1}, M=1.8 \times 10^{-25} \mathrm{~kg}$ and $J=6-12 \mathrm{~cm}^{-1}$. The phonon cutoff frequency is equal to $\Omega_{c}=96.86 \mathrm{~cm}^{-1}$ indicating that the nonadiabatic limit is reached $\left(\Omega_{c}>4 J\right)$. The exciton-phonon coupling strength is fixed to $E_{B}=0.21$ $\mathrm{cm}^{-1}$. At time $t=0$, a coherence is created on the side site $m_{0}=1$ and the initial conditions satisfy $\Psi_{m}(t=0)=\delta_{m 1}$.

Information about the coherence transfer is displayed in figure 1 for $T=100 \mathrm{~K}$ (figure 1(a)) and $T=300 \mathrm{~K}$ (figure 1(b)). For a lattice containing $N$ sites, figure 1 shows the maximum value of the transmitted coherence, i.e. $t_{N}=\operatorname{Max}\left[\left|\Psi_{N}(t)\right|\right]$, which occurs over an observation time scale equal to 1000 ps. At low temperature (figure $1(\mathrm{a})$ ), $t_{N}$ decreases almost linearly with the lattice size. It typically scales as $t_{N} \approx 1-0.02 N \forall J$, giving rise to a high fidelity transfer. At low temperature the coherence properties are mainly governed by the exciton Hamiltonian. Therefore, the discrete nature of the energy spectrum and the symmetry between the side sites favor the occurrence of quantum recurrences. For specific revival times, the exciton reaches a quantum state that resembles the copy of the initial state localized on the side site $m=N$. Consequently, the maximum of the transmitted coherence occurs after the exciton has experienced several reflections. For instance, for $J=6 \mathrm{~cm}^{-1}$ and $N=10, t_{N}=0.95$ is very close to unity. The maximum takes place at $t=742$ ps. Note that a fully different behavior was observed in an infinite lattice [10] since dephasing-limited coherent motion yields a strong localization of the coherence that decreases exponentially with distance.

At high temperature (figure 1(b)), the transmitted coherence behaves differently and a critical size $N^{*} \approx 10$ discriminates between two regimes. When $N<N^{*}$, $t_{N}$ still decreases almost linearly with $N, \forall J$. However, when $N>N^{*}, t_{N}$ exhibits pronounced peaks for specific $N$ values. The amplitude of these peaks is close to or larger than unity and their position depends on the hopping constant. For instance, for $J=12 \mathrm{~cm}^{-1}$, a peak whose amplitude is equal to 1.55 takes place for $N=11$. It corresponds to a maximum of the transmitted coherence that occurs at $t=948 \mathrm{ps}$. Similarly, for $J=8 \mathrm{~cm}^{-1}$, a peak whose amplitude is equal to 1.05 is observed for $N=13$ and the corresponding revival time is equal to $870 \mathrm{ps.} \mathrm{Consequently,} \mathrm{since}$ $\Psi_{N}(t)$ is an effective probability amplitude, these peaks reveal the failure of the $\mathrm{TCL}_{2}$ - 

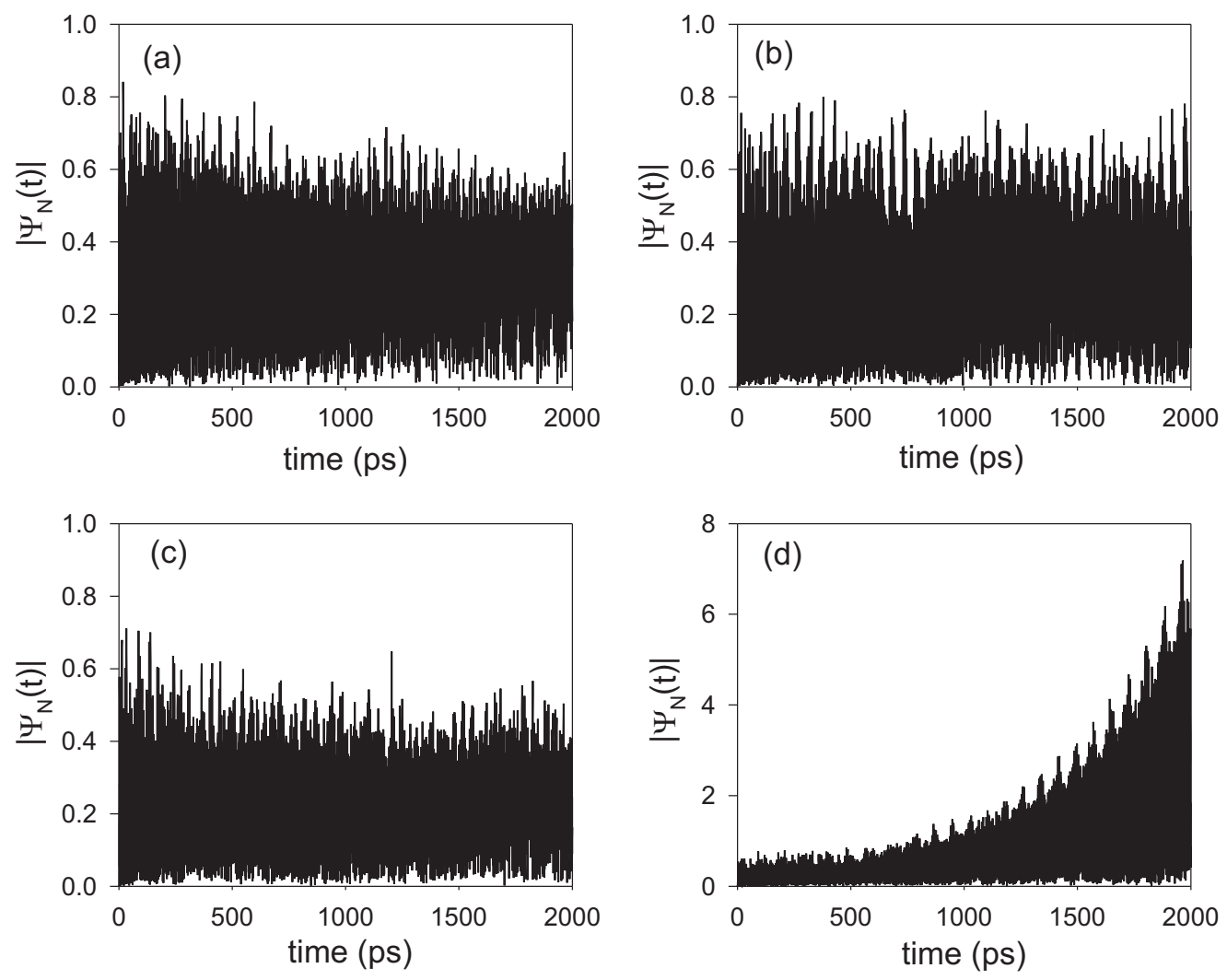

Figure 3. Time evolution of $\left|\Psi_{N}(t)\right|$ for $J=12 \mathrm{~cm}^{-1}$ and for (a) $N=8$, (b) $N=9$, (c) $N=10$ and (d) $N=11$.

GME to provide results with well-established physical meaning. Note that peaks have been observed at low temperature $(T=100 \mathrm{~K})$ but for a larger value of the excitonphonon coupling strength $E_{B}=0.5 \mathrm{~cm}^{-1}$ (not drawn). Therefore, although figure 1(a) seems to suggest that the confinement enhances the fidelity of the coherence transfer, the validity of the results is questionable.

According to the previous results, it seems that the $\mathrm{TCL}_{2}$-GME breakdown occurs for a rather strong exciton-phonon interaction, i.e. either at high temperature or for a strong coupling strength. However, a surprising effect is illustrated in figure 2 that shows the temperature dependence of $t_{N}$ for $J=12 \mathrm{~cm}^{-1}$. When $N=11, t_{N}$ remains lower than unity provided that $T<280 \mathrm{~K}$. Then, it exceeds unity over the temperature range $T \approx 290-350 \mathrm{~K}$, indicating the failure of the $\mathrm{TCL}_{2}$-GME. However, as the temperature increases, this breakdown seems to disappear since $t_{N}$ becomes lower than unity again. By contrast, for $N=12$ and over the temperature range displayed on the figure, $t_{N}$ exceeds unity at high temperature, i.e. when $T>400 \mathrm{~K}$.

To clarify the meaning of the previous features, the time evolution of $\left|\Psi_{N}(t)\right|$ is shown in figure 3 for $J=12 \mathrm{~cm}^{-1}, T=300 \mathrm{~K}$ and for different $N$ values. In the short time limit, the behavior of the coherence is qualitatively size independent. Initially equal to zero, it reaches a first maximum for a time $t_{L}$ that increases linearly with the lattice size. This maximum characterizes a direct exciton transfer 

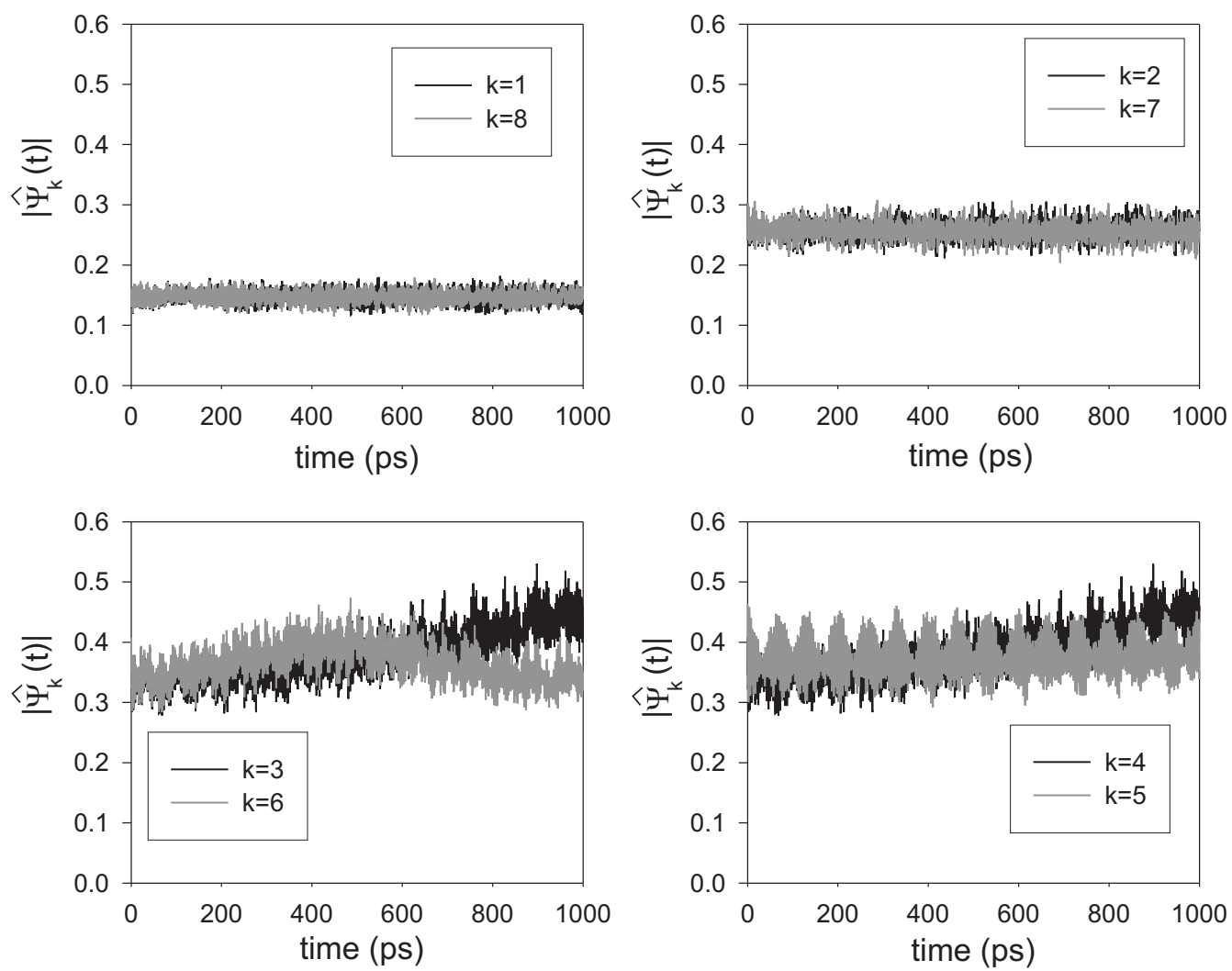

Figure 4. Time evolution of $\left|\hat{\Psi}_{k}(t)\right|$ for $J=12 \mathrm{~cm}^{-1}, T=300 \mathrm{~K}$ and $N=8$

between the two lattice sides according to a velocity of about $v=2 J$. Then, due to the confinement, $\left|\Psi_{N}(t)\right|$ does not vanish but it exhibits peaks which are almost periodically distributed. These peaks define quantum recurrences that occur at classical revival times $t_{k} \approx t_{L}+2 k L / v$, with $k=0,1,2 \ldots$ [41]. They describe situations in which the exciton quantum state resembles the copy of the initial state localized on the site $m=N$. Over a longer time scale, this scenario still remains valid and it yields a quite good understanding of the coherence behavior for $N=8,9$ and 10. Note that the strength of the recurrences slightly decreases as time increases. By contrast, for $N=11$ (figure 3(d)), a fully different behavior occurs. Although, the transmitted coherence supports quantum recurrences, it irreversibly increases with time and it exceeds unity when $t$ becomes longer than $800 \mathrm{ps}$. The coherence follows a slowly varying exponential growth which indicates the occurrence of an instability in the $\mathrm{TCL}_{2}$-GME.

To gain further insight into the origin of the instabilities, let us now consider the behavior of the coherences in the eigenbasis. To proceed, the time evolution of $\left|\hat{\Psi}_{k}(t)\right|$ is displayed in figure 4 for $J=12 \mathrm{~cm}^{-1}, T=300 \mathrm{~K}$ and $N=8$, i.e. a situation for which $t_{N}<1$ (see figures 1 (b) and 3(a)). When $\Delta H=0$, $\hat{\Psi}_{k}(t)=\hat{\Psi}_{k}(0) \exp \left(-i \omega_{k} t\right)$, with $\hat{\Psi}_{k}(0)=\sqrt{2 / L} \sin \left(k m_{0} \pi / L\right)$. The coherences evolve in pairs since $\hat{\Psi}_{k}(0)=\hat{\Psi}_{L-k}(0)$ and their modulus remains time independent, i.e. 
$\left|\hat{\Psi}_{k}(t)\right|=\left|\hat{\Psi}_{k}(0)\right|, \forall t$. When the exciton-phonon coupling is turned on, the coherences still evolve in pairs. Nevertheless, $\left|\hat{\Psi}_{k}(t)\right|$ is no longer time independent and, over $1000 \mathrm{ps}$, it shows high frequency small amplitude oscillations around its initial value. Note that the coherences connected to eigenstates close to the band center are more sensitive to the phonon bath. Nevertheless, over the time scale shown in figure 4, $\sum_{k}\left|\hat{\Psi}_{k}(t)\right|^{2}<1$. Initially equal to unity, the sum develops high frequency small amplitude oscillations around a mean value of about 0.75 . Therefore, no fact allows us to reject the probability amplitude interpretation of the coherences and the results displayed in figure 4 seem to indicate that a certain excitonic coherence remains in spite of the disturbance induced by the phonon bath.

As illustrated in figure 5, a different behavior takes place when $N=11$. In the short time limit, i.e. over $100 \mathrm{ps}$, the coherences evolve in pairs and they exhibit small amplitude oscillations around their initial value. Then, as time increases, a small disturbance affects the coherences associated to $k=1, N$ and $L / 2$. Note that coherences connected to $k=2,3,4,8,9$ and 10 are not significantly perturbed over the time scale shown in figure 5. By contrast, the coherences associated to $k=5$ and $k=7$ follow a slowly varying function that irreversibly increases exponentially and that supports high frequency small amplitude oscillations. The amplitude of these oscillations increases at time increases and the coherences exceed unity when $t$ becomes longer than $800 \mathrm{ps}$. This feature is the signature of an instability which yields the peak observed in figure 1(b) as well as to the divergence of the transmitted coherence displayed in figure 3(d). It reveals that the instability initially affects specific coherences that develop an exponential growth. However, as time increases, the instability propagates to the remaining coherences that finally diverge in the long time limit (not drawn in figure 5).

At this step, let us mention that the previous observations have a rather general character. Indeed, we have verified that the peaks displayed in figure 1(b) correspond to instabilities that involve an exponential growth of specific coherences in the eigenbasis, this growth being the precursor for the divergence of the remaining coherences. In most situations displayed in figure 1(b), the specific coherences are connected to eigenstates located in the neighborhood of the band center.

As shown in equation (11), the $\mathrm{TCL}_{2}$-GME breakdown results form the excitonphonon coupling that is encoded in the relaxation operator $\mathcal{J}(t)$. In a finite size lattice, the confinement is responsible for time recurrences which prevent $\mathcal{J}(t)$ to converge in the long time limit. As a result, the elements $\mathcal{J}\left(k_{1}, k_{2}, t\right)$ behave as almost periodic functions whose frequencies involve the difference between excitonic Bohr frequencies and phonon frequencies (see equations (12) and (13)). This specific time evolution yields an apparent randomness of $\mathcal{J}\left(k_{1}, k_{2}, t\right)$ whose properties can be investigated by performing a statistical analysis [41]. To proceed, $\mathcal{J}\left(k_{1}, k_{2}, t\right)$ can be viewed as a realization of a random variable $\mathcal{J}_{k_{1} k_{2}}$. Over a sufficiently long time scale, the curve $\mathcal{J}\left(k_{1}, k_{2}, t\right)$ defines a sample over which statistical quantities can be computed in an empirical way, i.e. without deploying a statistical model.

Within this statistical point of view, distribution functions provide information about the coupling strength between the coherences. To illustrate this feature, let us focus our attention on the coherences connected to $k=5$ and $k=7$ that exhibit an exponential growth for $J=12 \mathrm{~cm}^{-1}, T=300 \mathrm{~K}$ and $N=11$. Figure 6 displays the distribution function $g(\gamma)$ associated to $\gamma=\operatorname{Re} \mathcal{J}(5,7, t)$. The distribution shows a continuous band centered on the origin and whose width is about $1.7 \mathrm{~cm}^{-2}, \forall N$. It exhibits two peaks for $\gamma \approx \pm 0.35 \mathrm{~cm}^{-2}$. Although these peaks are slightly more 
Parametric resonance-induced time-convolutionless master equation breakdown
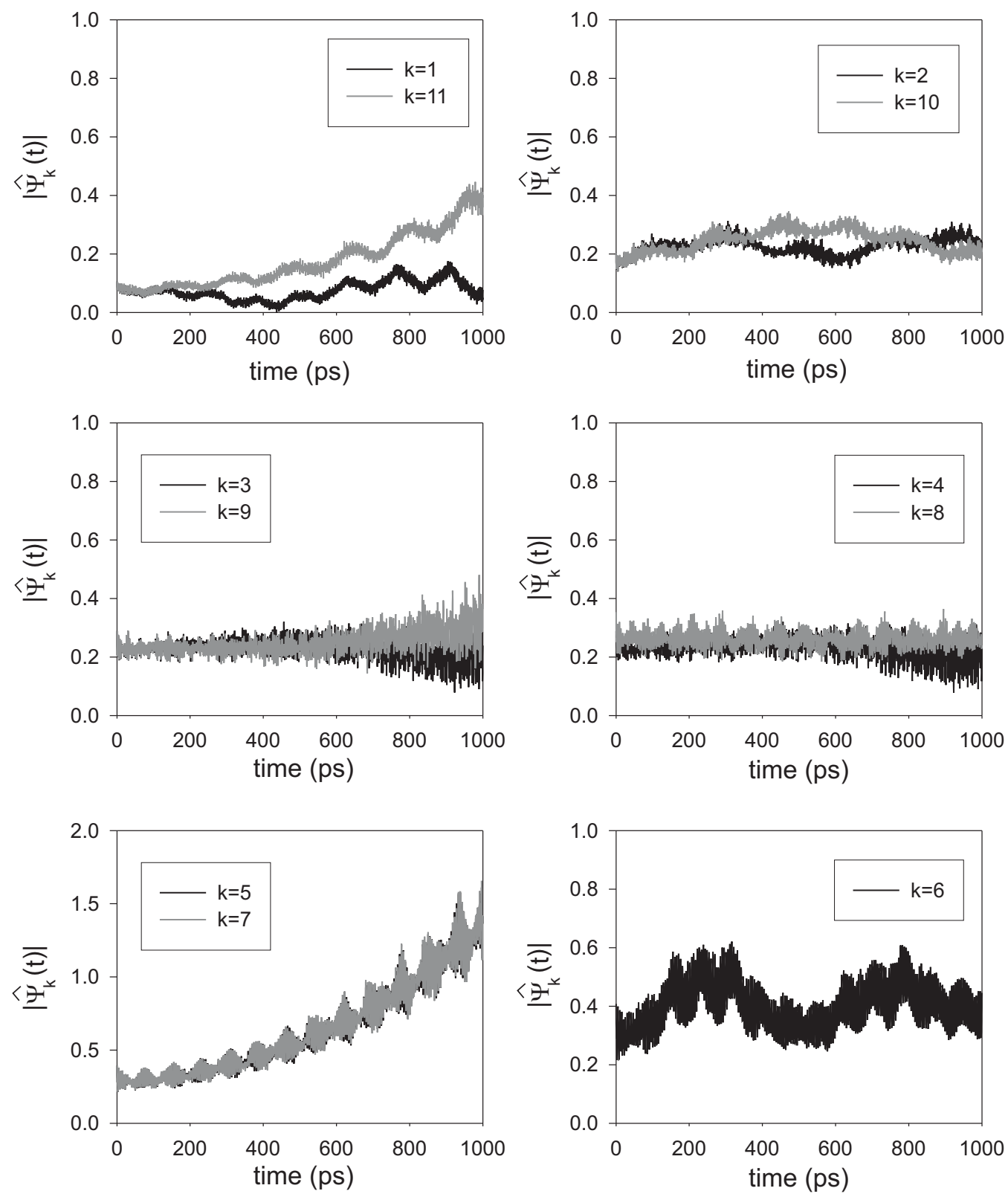

Figure 5. Time evolution of $\left|\hat{\Psi}_{k}(t)\right|$ for $J=12 \mathrm{~cm}^{-1}, T=300 \mathrm{~K}$ and $N=11$ 

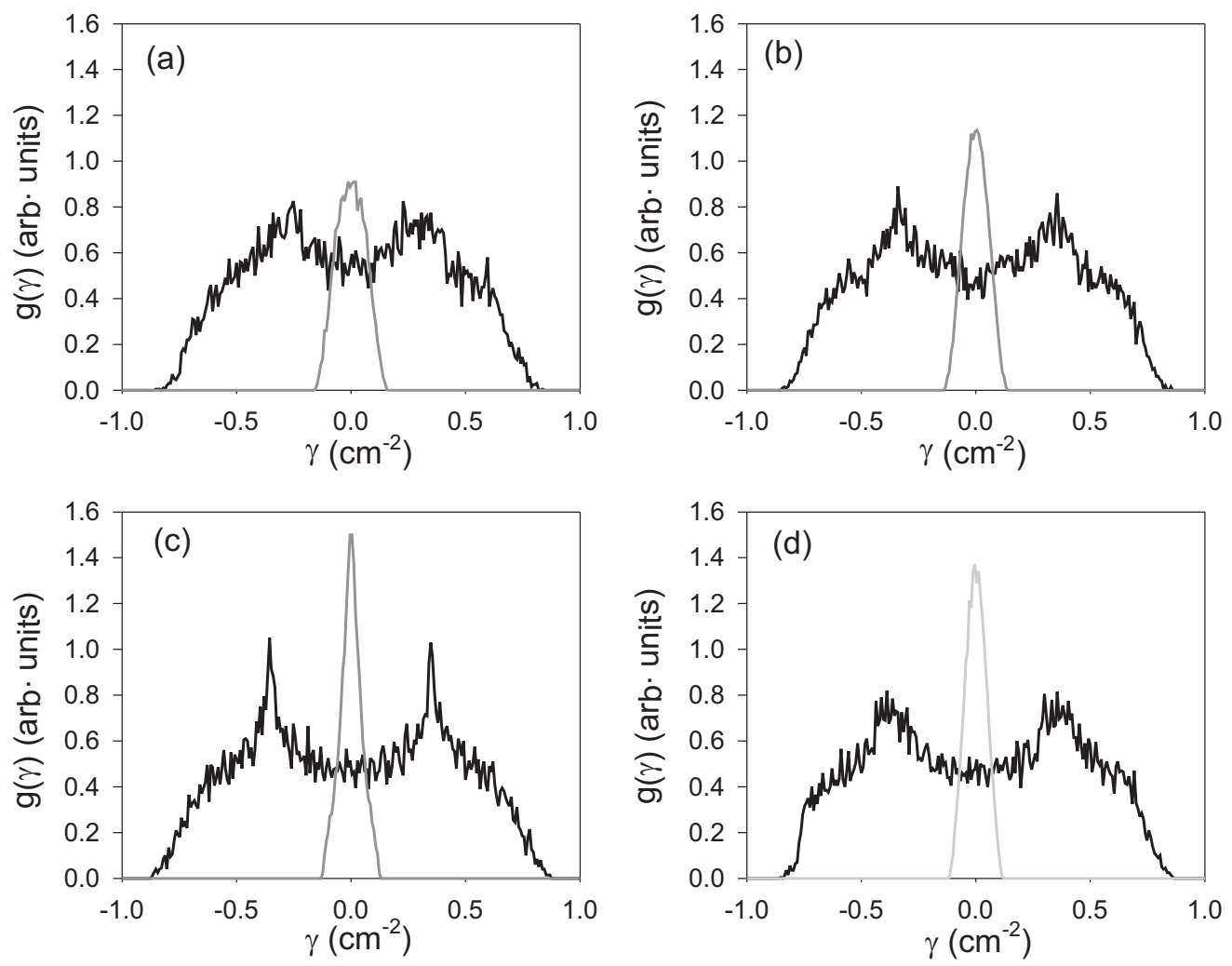

Figure 6. Distribution function for $\gamma=\operatorname{Re} \mathcal{J}(5,7, t)$ (black lines) for $J=12$ $\mathrm{cm}^{-1}, T=300 \mathrm{~K}$ and for (a) $N=9$, (b) $N=10$, (c) $N=11$ and (d) $N=12$. Gray lines represent the distribution when the coupling with the phonon mode $p=1$ is removed.

pronounced for $N=11$, the distribution is almost size independent. However, figure 6 reveals that the coupling with the phonon mode $p=1$ is the dominant contribution to $\mathcal{J}(t)$. Indeed, when this mode is removed from $\mathcal{J}(t)$, the distribution drastically narrows (gray lines). The peaks disappear and the distribution exhibits an almost uniform band whose width is equal to $0.28, \forall N$. By studying other relaxation operator elements, we have verified that this feature is rather general. More precisely, the phonon mode $p=1$ has been identified as the mode responsible for the occurrence of instabilities. To check this statement, the simulation of equation (11) has been carried out by switching off the influence of the mode $p=1$ in $\mathcal{J}(t)$. Doing so, we have observed the disappearance of any exponential growth.

Nevertheless, as suggested by the results displayed in figure 6 , the coupling strength with the phonon mode $p=1$ is not the key ingredient involved in the occurrence of instabilities. The main reason is that the coupling distribution is basically size independent whereas unstable evolutions arise for specific lattice size. In fact, the instabilities originate in the almost periodic nature of $\mathcal{J}(t)$. When the bath reduces to the mode $p=1$, the $\mathrm{TCL}_{2}$-GME is a linear system of differential equations whose time dependent coefficients evolve almost periodically. In the language of the differential equations, $\mathcal{J}(t)$ plays the role of a parameter that provides an almost 
periodic modulation of the exciton Hamiltonian $H_{A}$. In that context, one may expect the occurrence of parametric resonances when specific frequencies of $\mathcal{J}(t)$ approach specific excitonic eigenfrequencies $[52,53]$. To clarify this point, let us consider the socalled linear parametric oscillator. A parametric oscillator is a modification of a simple harmonic oscillator in which the natural frequency $\omega_{0}$ supports a periodic modulation, i.e. $\omega_{0}^{2} \rightarrow \omega_{0}^{2}+b \cos (\Omega t)$, where $b$ and $\Omega$ stand for the modulation strength and the modulation frequency, respectively. The dynamics being described by Mathieu-type equations [54], there are parameter values for which the trivial solution is unstable in the sense that a small disturbance will result in an unbounded exponential growth. Such an instability takes place if the parametric resonance condition $\Omega=2 \omega_{0} / n$ is fulfilled, the integer $n$ defining the order of the resonance.

In our situation, the coherences $\hat{\Psi}_{k}(t)$ can be viewed as the coordinates of harmonic oscillators whose natural frequencies are the eigenfrequencies $\omega_{k}$ whereas $\mathcal{J}(t)$ plays the role of the periodic modulation. Restricting our attention to the phonon mode $p=1$, the selection rules (8) show that the bath induces scattering from $|k\rangle$ to $|k \pm 1\rangle$. As a result, a coupling takes place between the coherences $\hat{\Psi}_{k}(t)$ and $\hat{\Psi}_{k \pm 2}(t)$ mediated by the elements $\mathcal{J}(k, k \pm 2, t)$. According to (13), these elements involve the frequencies $\omega_{k \pm 2}-\omega_{k \pm 1} \pm \Omega_{1}$. Therefore, one may expect an unstable evolution of both $\hat{\Psi}_{k}(t)$ and $\hat{\Psi}_{k \pm 2}(t)$ if a parametric resonance either defined by $\left|\omega_{k \pm 2}-\omega_{k \pm 1} \pm \Omega_{1}\right|=2 \omega_{k} / n$ or $\left|\omega_{k \pm 2}-\omega_{k \pm 1} \pm \Omega_{1}\right|=2 \omega_{k \pm 2} / n$ takes place.

To illustrate this feature, figure 7 displays the $k$ dependence of specific energy differences for $J=12 \mathrm{~cm}^{-1}$ and $N=11$. It shows that a second order parametric resonance $(n=2)$ affects the coherences $\hat{\Psi}_{5}(t)$ and $\hat{\Psi}_{7}(t)$, these coherences interacting through the relaxation operator element $\mathcal{J}(5,7, t)$. This resonance is thus responsible for the exponential growth of $\left|\hat{\Psi}_{5}(t)\right|$ and $\left|\hat{\Psi}_{7}(t)\right|$, as observed in figure 5. Of course, we have verified that parametric resonances can be invoked to explain the various peaks observed in figure 1 (b). For instance, for $J=8 \mathrm{~cm}^{-1}$ and $N=13$, a first order parametric resonance affects $\hat{\Psi}_{6}(t)$ and $\hat{\Psi}_{8}(t)$ which effectively exhibit an exponential growth at $T=300 \mathrm{~K}$. Similarly, for $J=10 \mathrm{~cm}^{-1}$ and $N=16$, two second order parametric resonances have been identified. The first resonance affects $\hat{\Psi}_{9}(t)$ and $\hat{\Psi}_{7}(t)$ whereas the second resonance acts on $\hat{\Psi}_{8}(t)$ and $\hat{\Psi}_{10}(t)$. We have checked that these four coherences diverge and thus produce a peak in the curve displayed in figure $1(\mathrm{~b})$.

At this step, let us consider in more details the situation $J=12 \mathrm{~cm}^{-1}$, $N=11$ and $T=300 \mathrm{~K}$ for which $\hat{\Psi}_{5}(t)$ and $\hat{\Psi}_{7}(t)$ diverge. Because of their exponential growth, we have verified that these coherences are decoupled from the other coherences. As a result, their dynamics is well accounted by the restriction of the GME to the subspace generated by the reduced basis $\{|k=5\rangle,|k=7\rangle\}$. Due to the symmetry of the exciton energy spectrum with respect to the band center, one obtains $\omega_{0}=\left(\omega_{5}-\omega_{6}\right)=-\left(\omega_{7}-\omega_{6}\right)=6.21 \mathrm{~cm}^{-1}$ and $\omega_{0} \approx\left(\omega_{4}-\omega_{5}\right)=-\left(\omega_{8}-\omega_{7}\right)$. For the mode $p=1$, the phonon frequency is fixed to $\Omega_{1}=12.64 \mathrm{~cm}^{-1}$. At high temperature, the relaxation operator (12) is approximated by disregarding negligible contributions. For instance, $\mathcal{J}(5,5, t)$ involves the term $F_{145}(t)$ (equation $(13)$ ) that exhibits two contributions. The contribution whose denominator is equal to $\Omega_{1}-\omega_{0}$ is kept whereas the contribution whose denominator involves $\Omega_{1}+\omega_{0}$ is neglected. By generalizing this procedure, the relaxation operator is finally expressed as

$$
\begin{aligned}
\mathcal{J}(5,5, t) & =\mathcal{J}(7,7, t)=2 \operatorname{Re} \eta(t) \\
\mathcal{J}(5,7, t) & =\mathcal{J}^{*}(7,5, t)=\eta(t)
\end{aligned}
$$




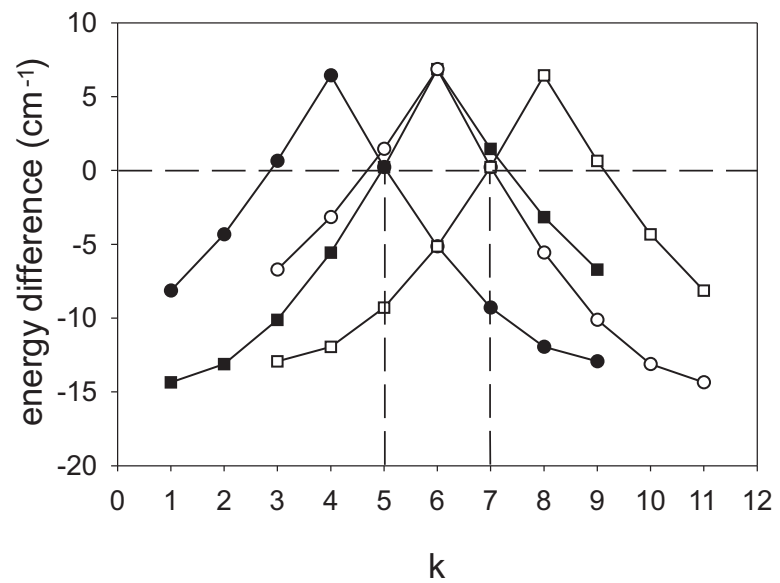

Figure 7. $k$ dependence of the energy differences $\left|\omega_{k+2}-\omega_{k+1}+\Omega_{1}\right|-\omega_{k}$ (full squares), $\left|\omega_{k+2}-\omega_{k+1}+\Omega_{1}\right|-\omega_{k+2}$ (full circles), $\left|\omega_{k-2}-\omega_{k-1}-\Omega_{1}\right|-\omega_{k}$ (open circles) and $\left|\omega_{k-2}-\omega_{k-1}-\Omega_{1}\right|-\omega_{k-2}$ (open squares), for $J=12 \mathrm{~cm}^{-1}$ and $N=11$.

where $\eta(t)=i \epsilon(1-\exp (i \Omega t))$, with $\epsilon=E_{B} k_{B} T / L \Omega$, is a periodic function with frequency $\Omega=\Omega_{1}-\omega_{0}$. Consequently, performing the change of variables $\hat{\Psi}_{k}(t) \rightarrow$ $\hat{\Psi}_{k}(t) \exp \left(-i e_{0} t\right)$, the reduced GME is written as

$$
\begin{aligned}
& \frac{d}{d t} \hat{\Psi}_{5}(t) \approx-i \omega_{0} \hat{\Psi}_{5}(t)-2 \operatorname{Re}(\eta(t)) \hat{\Psi}_{5}(t)-\eta(t) \hat{\Psi}_{7}(t) \\
& \frac{d}{d t} \hat{\Psi}_{7}(t) \approx+i \omega_{0} \hat{\Psi}_{7}(t)-2 \operatorname{Re}(\eta(t)) \hat{\Psi}_{7}(t)-\eta^{*}(t) \hat{\Psi}_{5}(t)
\end{aligned}
$$

The coherence dynamics is thus described by a linear system of differential equations with periodic coefficients whose period is equal to $T=2 \pi / \Omega$. As a result, the stability of the solutions can be studied within the frame of the Floquet's theory [55]. According to this theory, the solutions of (15) behave as $\hat{\Psi}_{k}(t)=\exp (\mu t) f_{k}(t)$ where $f_{k}(t)$ is a $T$-periodic function and where $\mu$ defines the so-called Floquet exponent. Equation (15) is characterized by two Floquet exponents $\mu_{i}$ that are related to the characteristic multipliers $\rho_{i}$ according to the relation $\rho_{i}=\exp \left(\mu_{i} T\right)$, for $i=1,2$. The characteristic multipliers are the eigenvalues of the monodromy matrix that corresponds to the evolution operator (fundamental matrix) connected to (15) evaluated at time $t=T$. Consequently, three regimes occur depending on the value of the Floquet exponents. If $\operatorname{Re}(\mu)<0(|\rho|<1)$, the solution tends to zero in the long time limit. By contrast, if $\operatorname{Re}(\mu)=0(|\rho|=1)$, a pseudo-periodic solution arises. Note that if $\rho= \pm 1$, the solution is periodic with period $T$. Finally, if $\operatorname{Re}(\mu)>0$ $(|\rho|>1)$, an unstable solution occurs giving rise to an exponential growth.

In that context, the simulation of (15) has been carried out over a period $T$ to evaluate the monodromy matrix and to determine the Floquet exponents. This procedure allows us to define the stability diagram in the parameter space $(\epsilon, \Omega)$. As illustrated in figure 8 , this diagram shows instability domains, i.e. the striped regions, that form tongues which fall down to zero. These so-called Floquet tongues emanate from the parametric resonances $\left(\Omega / \omega_{0} \approx 2 / n, \epsilon=0\right)$, with $n$ a positive integer, where they exhibit more or less sharped tips. Near a resonance, an infinitesimal disturbance destabilizes the dynamics and it gives rise to the exponential growth of the coherences. 


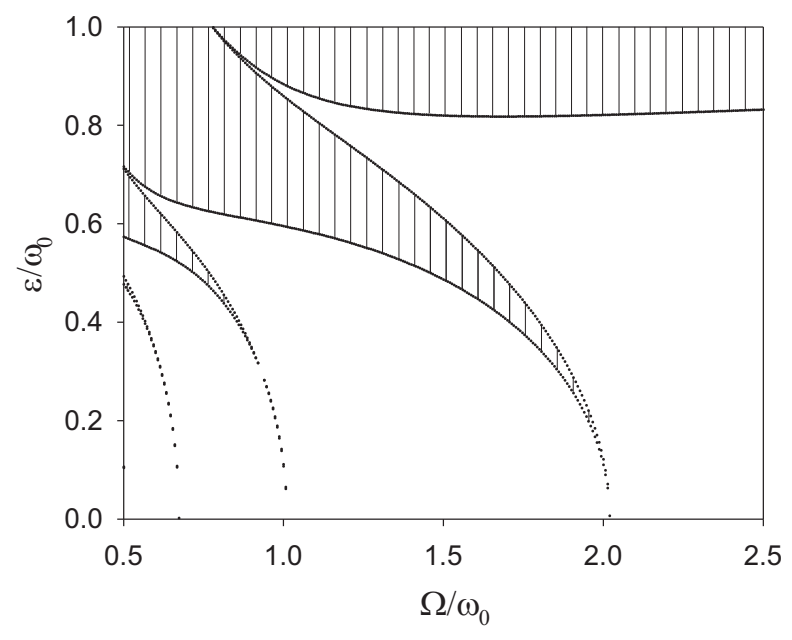

Figure 8. Stability diagram in the parameter space for equation (15) (see the text). Striped regions correspond to unstable solutions.

Note that at $T=300 \mathrm{~K}$, one obtains $\Omega / \omega_{0}=1.035$ and $\epsilon / \omega_{0}=0.093$ indicating that the divergence of the coherences originates in a second order parametric resonance, as observed in figure 7. Moreover, since $\epsilon$ is proportional to the temperature, increasing the temperature amounts to follow a straight line in the stability diagram for a fixed $\Omega$ value. Doing so, one successively crosses stable and unstable regions. For instance, for $\Omega=1.5 \omega_{0}$, stable solutions arise provided that $\epsilon<0.48$. Then, an unstable region is covered when $0.48<\epsilon<0.61$ but a stable domain recurs when $\epsilon$ exceeds 0.61 . Finally, unstable solutions take place provided that $\epsilon>0.81$. This feature explain why instabilities occur over a temperature range, whereas stable solutions appear outside this range, as illustrated in figure 2. Note that the occurrence of instabilities results form the coupling between the coherences mediated by the non diagonal elements of the relaxation operator. When the diagonal part of $\mathcal{J}(t)$ is removed form equation (15), one obtains a rather similar stability diagram with the same parametric resonances.

To conclude, let us mention that the prediction provided by the $\mathrm{TCL}_{2}$-GME has clearly no physical meaning when a parametric resonance takes place. By contrast, outside the instability domains, no fact allows us to reject the wave function interpretation of the coherences and our results seem to indicate that the confinement enhances the fidelity of the transfer (see figures 1(a), 3(a), 3(b) and 3(c)). However, the sensitivity of the GME to the model parameters makes this statement questionable. To clarify this point, an exact diagonalization has been carried out when the phonon bath reduced to the single mode $p=1$. In doing so, we have observed that the $\mathrm{TCL}_{2}$ GME yields quite good results in the very short time limit, only. Over intermediate and long time scales, the $\mathrm{TCL}_{2}$-GME breaks down although no divergence occurs. It always overestimates the degree of the coherence when compared with exact calculations. Strictly speaking, this feature does not originate in the perturbative treatment of the exciton-phonon coupling. It results from the Born approximation in which the relaxation operator depends on time through memory functions. These memory functions characterize a free evolution of the phonons that form a thermal bath insensitive to the exciton dynamics. In that context, we have verified that 
standard canonical second order perturbation theory provides a time evolution in good agreements with the exact diagonalization method, even in the long time limit. The calculations have been carried out by using a dressed eigenbasis that mixes both exciton and phonon degrees of freedom. Correlations are thus taken into account although a second order expansion with respect to the exciton-phonon coupling is performed.

\section{Conclusion}

In the present paper, a detailed analysis was performed to show that the $\mathrm{TCL}_{2}$-GME fails to describe the dynamics of an exciton coupled with a phonon bath in a finite size lattice. Special attention has been paid to characterize the properties of the excitonic coherences. They define specific elements of the exciton RDM that measure the ability of the exciton to develop superimpositions involving the vacuum and the one-exciton states. These coherences play a key role in understanding various phenomenon since they provide information about the linear response of the exciton and studying their propagation is a key step to understand quantum state transfer in open systems.

In the excitonic eigenbasis, we have shown that the coherences behave as wave functions whose dynamics is governed by a time-dependent effective Hamiltonian. The influence of the phonon bath is encoded in a time-dependent relaxation operator that provides to the effective Hamiltonian its non hermitian nature. Due to the confinement, quantum recurrences arise so that the relaxation operator does not converge in the long time limit. A non-Markovian regime takes place and the relaxation operator exhibits an almost periodic nature whose main contribution results from the coupling with the phonon mode $p=1$. Consequently, the $\mathrm{TCL}_{2}$-GME reduces to a linear system of differential equations with almost periodic coefficients. In accordance with the Floquet theory, we have shown that unstable solutions emerge for particular values of the model parameters. Such instabilities originate in parametric resonances that involve specific frequencies of the relaxation operator and specific excitonic eigenfrequencies. They give rise to an exponential growth of the coherences attached to eigenstates located in the neighborhood of the band center. These initial instabilities propagate to the remaining coherences that finally diverge in the long time limit leading to the $\mathrm{TCL}_{2}$-GME breakdown. Moreover, if the prediction provided by the $\mathrm{TCL}_{2}$-GME has clearly no physical meaning when a parametric resonance occurs, we have shown that this method overestimates the degree of the excitonic coherence when no resonance is observed. Consequently, whatever the situation, the $\mathrm{TCL}_{2}$ GME fails to provide good results over both intermediate and long time scales. These features originate in the Born approximation in which correlations between the exciton and the phonons are neglected during the propagation. Therefore, to overcome this problem, both exact diagonalization and canonical perturbation theory will be applied in forthcoming works to investigate again the exciton-phonon dynamics in a finite size lattice.

\section{References}

[1] Edler J, Pfister R, Pouthier V, Falvo C and Hamm P 2004 Phys. Rev. Lett. 93106405

[2] Tsivlin D and May V 2007 Chem. Phys. 338150

[3] Ivic Z, Kostic D, Przulj Z and Kapor D 1997 J. Phys.: Condens. Matter 9413

[4] Cruzeiro L 2005 J. Phys.: Condens. Matter 177833

[5] Pouthier V 2009 J. Phys.: Condens. Matter 21185404 
[6] Bodis P, Schwartz E, Koepf M, Cornelissen J J L M, Rowan A E, Nolte R J M and Woutersen S 2009 J. Chem. Phys. 131124503

[7] Bittner E R, Goj A M and Burghardt I 2010 Chem. Phys. 370137

[8] Pouthier V and Tsybin Y O 2008 J. Chem. Phys. 129095106

[9] Pouthier V 2010 J. Chem. Phys. 132035106.

[10] Pouthier V 2010 J. Phys.: Condens. Matter 22255601.

[11] Curzeiro L 2009 J. Biol. Phys. 3543

[12] Renger Th and Marcus R A 2002 J. Chem. Phys. 1169997

[13] Schroder M, Kleinekathofer U and Schreiber M 2006 J. Chem. Phys. 124084903

[14] Renger T, May V, and Kuhn O 2001 Phys. Rep. 343137

[15] Sundstrom V 2000 Prog. in Quantum Electron. 24187

[16] Meier T, Zhao Y, Chernyak V, and Mukamel S 1997 J. Chem. Phys. 1073876

[17] Persson B N J 1992 Phys. Rev. B 4612701

[18] Bonn M, Hess C and Wolf M 2001 J. Chem. Phys. 1157725

[19] Pouthier V, Light J C and Girardet C 2001 J. Chem. Phys. 1144955

[20] Jakob P 2001 J. Chem. Phys. 1143692

[21] Pouthier V 2003 J. Chem. Phys. 1183736

[22] Roeterdink W G, Berg O and Bonn M 2004 J. Chem . Phys. 12110174

[23] Pouthier V 2005 Phys. Rev. B 71115401

[24] Pouthier V and Girardet C 1999 Phys. Rev. B 6013800

[25] Fröhlich H 1954 Adv. Phys. 3325

[26] Holstein T 1959 Ann. Phys. (N.Y.) 8 325; 19598343

[27] Breuer H P and Petruccione F 2007 The Theory of Open Quantum Systems (New York: Oxford University Press)

[28] May V and Kuhn O 2000 Charge and Energy Transfer Dynamics in Molecular Systems (Berlin: Wiley-VCH Verlag)

[29] Schlosshauer M 2007 Decoherence and the Quantum-to-Classical Transition (Berlin: Spinger Verlag)

[30] Nakajima S 1958 Prog. Theor. Phys. 20948

[31] Zwanzig R 1960 J. Chem. Phys. 331338

[32] Shibata F, Takahashi Y and Hashitsume N 1977 J. Stat. Phys. 17171

[33] Uchiyama C and Shibata F 1999 Phys. Rev. E 602636

[34] Barnett S M and Stenholm S 2001 Phys. Rev. A 64033808

[35] Silbey R and Munn R W 1979 J. Chem. Phys. 722763

[36] Capek V and I. Barvik I 1987 J. Phys. C 201459

[37] Dolderer D and Wagner M 1998 J. Chem. Phys. 108261

[38] Pouthier V 2008 Phys. Rev. E 78061909

[39] Sanda F 2002 Czechoslovak Journal of Physics 52729

[40] Pouthier V 2009 Phys. Rev. B 80144304

[41] Pouthier V 2010 Phys. Rev. E 81031913

[42] Pouthier V 2007 Phys. Rev. E 75061910

[43] Pereverzev A and Bittner E R 2006 J. Chem. Phys. 125104906

[44] Breuer H P 2007 Phys. Rev. A 75022103

[45] Fischer J and Breuer H P 2007 Phys. Rev. A 76052119

[46] Ferraro E, Breuer H P, Napoli A, Jivulescu M A and Messina A 2008 Phys. Rev. B 78064309

[47] Willis C R and Picard R H 1974 Phys. Rev. A 91343

[48] Koide T 2002 Prog. Theo. Phys. 107525

[49] Linden O and May V 1998 Physica A 254411

[50] Hughes K H, Christ C D and Burghardt I 2009 J. Chem. Phys. 131024109

[51] Hughes K H, Christ C D and Burghardt I 2009 J. Chem. Phys. 131124108

[52] Kneubuhl F K 1997 Oscillations and Waves (Berlin: Springer-Verlag)

[53] Butikov E I 2004 Eur. J. Phys. 25535

[54] Bender C M and Orszag S A 1978 Advanced Mathematical Methods for Scientists and Engineers (New York: McGraw-Hill)

[55] Coddington E A and Levinson N 1955 Theory of Ordinary Differential Equations (New Yrok: McGraw-Hill) 